\title{
Microstructure of charged AdS black hole via $P-V$ criticality
}

\author{
Amin Dehyadegari, ${ }^{1}$ Ahmad Sheykhi, ${ }^{1,2,3, *}$ and Shao-Wen $\mathrm{Wei}^{4, \dagger}$ \\ ${ }^{1}$ Physics Department and Biruni Observatory, Shiraz University, Shiraz 71454, Iran \\ ${ }^{2}$ Research Institute for Astronomy and Astrophysics of Maragha (RIAAM), \\ P.O. Box 55134-441, Maragha, Iran \\ ${ }^{3}$ Max-Planck-Institute for Gravitational Physics (Albert-Einstein-Institute), 14476 Potsdam, Germany \\ ${ }^{4}$ Institute of Theoretical Physics \& Research Center of Gravitation, Lanzhou University, \\ Lanzhou 730000, People's Republic of China
}

(Received 9 July 2020; accepted 21 October 2020; published 6 November 2020)

\begin{abstract}
We suggest a new thermodynamic curvature, constructed via adiabatic compressibility, for examining the internal microstructure of charged black holes in an anti-de Sitter background. We analyze the microscopic properties of small-large phase transition of black holes with pressure and volume as the fluctuation variables. We observe that strong repulsive interactions dominate among the microstructures of near extremal small black holes, and the thermodynamic curvature diverges to positive infinity for the extremal black holes. At the critical point, however, thermodynamic curvature diverges to negative infinity.
\end{abstract}

DOI: 10.1103/PhysRevD.102.104013

\section{INTRODUCTION}

Phase transition is a fascinating phenomenon in black hole thermodynamics which has received considerable attention in recent years. This is mainly motivated by AdS/CFT duality, which states that there exists a correspondence between asymptotically anti-de Sitter (AdS) spacetime and the conformal field theory living on its boundary [1,2]. Significant interest has arisen for studying the phase transition of AdS black holes in an extended phase space in which the cosmological constant can be regarded as thermodynamic pressure which can vary [3,4]. In this viewpoint, the mass of black hole is identified as the enthalpy [5]. It was shown [4] that the four-dimensional charged AdS black hole demonstrates the first-order (discontinuous) and secondorder (continuous) phase transitions between the small and large black holes in an extended phase space. This phase transition is analogous to the van der Waals gas/liquid phase transition; thus, their critical exponents are the same as well. The investigation on the critical behavior of black holes in this context is often referred to as $P-V$ criticality and has been widely explored in the literature [6-15] and references therein. Some interesting phenomena have been observed in the extended phase space of black holes such as zeroth-order

\footnotetext{
*asheykhi@shirazu.ac.ir

†weishw@1zu.edu.cn
}

Published by the American Physical Society under the terms of the Creative Commons Attribution 4.0 International license. Further distribution of this work must maintain attribution to the author(s) and the published article's title, journal citation, and DOI. Open access publication funded by the Max Planck Society. phase transition [16] and reentrant phase transition [17] as well as triple critical point [18] as well as superfluid like phase transition [19]. More recently, a universality class of the critical behavior of AdS black holes in an extended phase space has been studied by a general approach without specifying the functional form of the spacetime metric [20].

An alternative approach to investigate critical behavior of black holes is to consider the electric charge $(Q)$ of the black hole as a thermodynamical variable while keeping the cosmological constant as a fixed parameter. From the physical point of view, the electric charge of black hole is a natural variable which can take on arbitrary values, and it affects the thermodynamic properties of AdS black hole. In this case, it was argued [21] that there exists a small-large black hole phase transition for the charged black hole in a fixed AdS background. It has been demonstrated [22] that this phase transition is physically conventional in an alternative phase space where the square of the electric charge $\left(Q^{2}\right)$ is viewed as an independent thermodynamic variable of the black hole system. In this perspective, the new thermodynamic response function correctly signifies stable and unstable regimes and the critical behavior of the black hole resembles van der Waals fluid, belonging to the same universality class [23]. Phase transition of black holes in an alternative phase space have been explored in different setups [24-26]. More recently, the authors of Ref. [27] investigated thermodynamic phase structure of Born-Infeld and charged dilaton [28] black holes in a fixed AdS spacetime by studying the behavior of specific heat.

The theory of covariant thermodynamic fluctuations provides a powerful geometric framework to study properties of underlying thermal system, completely from the 
thermodynamic viewpoint [29,30]. In this context, Ruppeiner defined the Riemannian metric on the equilibrium thermodynamic state space as the second derivatives of entropy. In his series of works [31-33], it has been confirmed that thermodynamic curvature (Ricci scalar) arising out of a such a metric is related to the microscopic interactions, where the thermodynamic curvature is positive (negative) for the repulsive (attractive) interaction. In addition, thermodynamic curvature diverges at the critical point for pure fluid systems. With regard to this approach, the microscopic behavior and phase transition of various kinds of black holes have been explored [34-37]. In all these works, thermodynamic curvature has a finite value at the critical point. Recently, a new normalized thermodynamic curvature was proposed to understand the microscopic behavior of a charged AdS black hole in an extended phase space where the temperature and volume are treated as fluctuating variables [38-40]. In this formalism, thermodynamic curvature is normalized with respect to the heat capacity at constant volume. It was shown that the microstructure of a small black hole has a weak repulsive interaction and the thermodynamic curvature goes to infinity at the critical point of phase transition. This method has recently been employed to determine the microstructure of several black hole systems [41-44].

In this paper, we offer a new thermodynamic curvature in the Ruppeiner geometry approach, which is constructed via the adiabatic compressibility, for examining the internal microstructure of charged AdS black holes in an extended phase space with fixed charge. Regarding the thermodynamic fluctuation metrics in Ruppeiner's formalism, we obtain a line element with the entropy and pressure parameter coordinates which differs from the approach given in Refs. [38,39]. Indeed, the authors of Refs. [38,39] investigate the microstructure of charged AdS black holes using the coordinates temperature and volume in the Ruppeiner geometry. Since the entropy only depends on the volume in the case of charged black holes, we express a line element in terms of the volume and pressure. Based on the vanishing of the adiabatic compressibility of the black holes, we propose a new normalized thermodynamic curvature. Then, we use the normalized thermodynamic curvature to probe the microscopic properties of small-large phase transition of black holes in an extended phase space. Our work differs from Refs. [38,39] in that they normalized the thermodynamic curvature by the heat capacity at constant volume, while here we normalize the thermodynamic curvature by the adiabatic compressibility. Besides, considering the pressure $P$ as a coordinate, the authors of Ref. [41] pointed out that the scalar curvature does not diverge at the critical point, which is not consistent with the observation of Ruppeiner's result. However, in our novel approach, the scalar curvature has no such problem. This also gives us a chance to examine the critical behavior of the scalar curvature. We shall observe that strong repulsive interactions dominate among the microstructures of small black holes where the thermodynamic curvature diverges to positive infinity. It is shown that the thermodynamic curvature diverges to negative infinity at the critical point.

The structure of the paper is laid out as follows. We begin in Sec. II by giving a brief review of the thermodynamics and critical behavior of the four-dimensional charged AdS black holes in the extended phase space. In Sec. III, we first introduce the Ruppeiner geometry and obtain the corresponding line element for a thermodynamic system in terms of the entropy and pressure. Then, we use the thermodynamic curvature to investigate in detail the microstructure of charged AdS black hole. Finally, we present some remarks in Sec. IV.

\section{THERMODYNAMICS AND PHASE TRANSITION OF CHARGED ADS BLACK HOLES}

We start with a brief review on the thermodynamics properties and $P-V$ criticality of a Reissner-Nordstrom (RN-)AdS black hole in an extended phase space. The action of Einstein-Maxwell theory in four-dimensional spacetime with a cosmological constant $(\Lambda)$ is

$$
I=\frac{1}{16 \pi} \int d^{4} x \sqrt{-g}\left(\mathcal{R}-2 \Lambda-F_{\mu \nu} F^{\mu \nu}\right),
$$

where $\mathcal{R}$ is the scalar Riemann curvature and $F_{\mu \nu}$ is the electromagnetic field strength that is defined as $F_{\mu \nu}=$ $\partial_{\mu} A_{\nu}-\partial_{\nu} A_{\mu}$ with the gauge field $A_{\mu}$. The negative cosmological constant $\Lambda$ is related to the AdS radius $L$ by the relation, $\Lambda=-3 / L^{2}$. In four dimensions, the line element of the spherically symmetric RN-AdS metric is given by [4]

$$
\begin{aligned}
& d s^{2}=-f(r) d t^{2}+\frac{d r^{2}}{f(r)}+r^{2} d \Omega^{2}, \\
& f(r)=1-\frac{2 M}{r}+\frac{Q^{2}}{r^{2}}+\frac{r^{2}}{L^{2}},
\end{aligned}
$$

where $d \Omega^{2}$ is the metric of the unit 2-sphere. Herein, the parameters $M$ and $Q$ are, respectively, the mass and charge of the black hole where the position of the black hole event horizon $\left(r_{+}\right)$is determined as a largest positive real root of $f\left(r_{+}\right)=0$. The only nonvanishing component of the electromagnetic field tensor is given by $F_{t r}=Q / r^{2}$.

The Hawking temperature of the RN-AdS black hole on an event horizon is obtained as [4]

$$
T=\frac{f^{\prime}\left(r_{+}\right)}{4 \pi}=\frac{1}{4 \pi r_{+}}\left(1+\frac{3 r_{+}^{2}}{L^{2}}-\frac{Q^{2}}{r_{+}^{2}}\right),
$$

and the entropy is

$$
S=\pi r_{+}^{2}
$$


By interpreting the cosmological constant as a thermodynamic pressure, $P=-\Lambda /(8 \pi)$, and its conjugate quantity as a black hole thermodynamic volume, $V=4 \pi r_{+}^{3} / 3$, the first law of black hole thermodynamics and the corresponding Smarr formula take the forms, respectively,

$$
\begin{gathered}
d M=T d S+V d P+\Phi d Q, \\
M=2 T S+\Phi Q-2 V P,
\end{gathered}
$$

where $\Phi=Q / r_{+}$is the electric potential measured with respect to the event horizon. In this consideration, the mass $(M)$ of the black hole is identified as the enthalpy. Also, the thermodynamic process is carried out in the extended phase space. It is worthwhile to mention that, according to Eq. (5) and the black hole thermodynamic volume formula, the entropy is only a function of area/volume, i.e., $S=S(V)$. This feature of the black hole will be used in the next section.

For the four-dimensional charged AdS black hole, the equation of state, $P=P(T, V)$, is obtained by using Eq. (4) as

$$
P=\frac{T}{2 r_{+}}-\frac{1}{8 \pi r_{+}^{2}}+\frac{Q^{2}}{8 \pi r_{+}^{4}}
$$

where $r_{+}=(3 V / 4 \pi)^{1 / 3}$. The behavior of isotherms in the $P-V$ diagram is shown in Fig. 1. We see that the critical point is an inflection point on the isotherm, which is characterized by

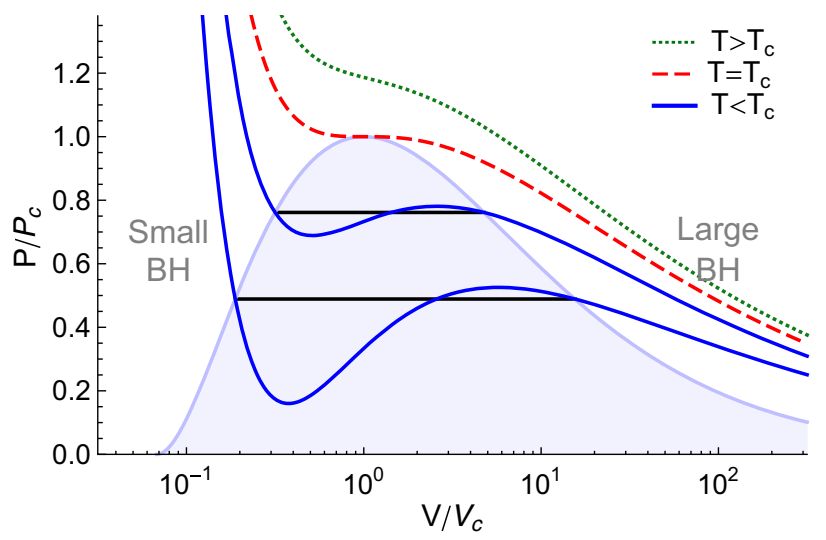

FIG. 1. $\quad P-V$ diagram of RN-AdS black holes. The region of the first-order phase transition is identified where the isobars (black horizontal lines) remedy the unstable regime by the Maxwell equal area law. The areas above and below the black isobar are equal to each other, which is not seen because of logarithmic scale on the horizontal axis. The critical point is marked by a black spot. Note the logarithmic scale on the horizontal axis.

$$
\left.\frac{\partial P}{\partial V}\right|_{T_{c}}=0,\left.\quad \frac{\partial^{2} P}{\partial V^{2}}\right|_{T_{c}}=0 .
$$

One obtains the critical quantities as

$$
T_{c}=\frac{\sqrt{6}}{18 \pi Q}, \quad P_{c}=\frac{1}{96 \pi Q^{2}}, \quad V_{c}=8 \sqrt{6} \pi Q^{3} .
$$

For $T<T_{c}$, an oscillating part of the isotherm denotes the unstable region where the isothermal compressibility is negative, i.e.,

$$
\kappa_{T}=-\left.\frac{1}{V} \frac{\partial V}{\partial P}\right|_{T}<0 .
$$

This instability is replaced by an isobar (the horizontal line) via the Maxwell equal area construction, $\oint V d P=0$, which means that there exists a first-order phase transition between the small black hole and large black hole. The small-large black hole transition region, determined by Maxwell construction, has the forms [45]

$$
\begin{aligned}
\tilde{T}^{2} & =\tilde{P}(3-\sqrt{\tilde{P}}) / 2, \\
\tilde{P} & =\frac{7+6 \tilde{V}^{2 / 3}-4 \sqrt{3+6 \tilde{V}^{2 / 3}}}{\tilde{V}^{4 / 3}},
\end{aligned}
$$

where the reduced thermodynamic variables are defined as

$$
\tilde{T}=\frac{T}{T_{c}}, \quad \tilde{P}=\frac{P}{P_{c}}, \quad \tilde{V}=\frac{V}{V_{c}} .
$$

It is worthwhile to note that the two phases of small and large black holes cannot be distinguished above the critical point. In the next section, we examine the behavior of charged black hole in the Ruppeiner geometry.

\section{RUPPEINER GEOMETRY}

In this section, we apply the concept of the Ruppeiner thermodynamic geometry as a useful tool to study the microscopic structure of charged AdS black holes. The Ruppeiner geometry arises from the Gaussian thermodynamic fluctuation theory, which is constructed on the thermodynamic state space [30]. In two dimensions, the Riemannian curvature scalar, $R$, (thermodynamic curvature) gives complete information about the Ruppeiner geometry, which is connected with the interparticle interaction in a thermodynamic system. Especially, the positive (negative) sign of the thermodynamic curvature indicates the repulsive (attractive) interaction, while $R=0$ corresponds to no interaction [31-33]. In the following, we first derive the thermodynamic fluctuation metric in the $(S, P)$ coordinates, where a thermodynamic potential is the enthalpy. Then, using the fact that the entropy of the charged AdS black hole only depends on the volume, we 
investigate the thermodynamic curvature of black hole through the $(P, V)$ plane.

\section{A. Ruppeiner metric}

Consider a thermodynamic system characterized by the entropy $(S)$, internal energy $(U)$, and volume $(V)$ such that the line element between two thermodynamic states is [30]

$$
\Delta l^{2}=g_{\mu \nu} \Delta x^{\mu} \Delta x^{\nu}
$$

where $x^{\mu}=(U, V)$ and the metric element $g_{\mu \nu}$ is given by

$$
g_{\mu \nu}=-\frac{\partial^{2} S}{\partial x^{\mu} \partial x^{\nu}} .
$$

In the entropy representation, the first law of thermodynamics for this system is expressed as

$$
d S=\frac{1}{T} d U+\frac{P}{T} d V
$$

where $T$ and $P$ are the temperature and pressure, respectively. Using the first law of thermodynamics, Eq. (13), the line element, Eq. (12), can be written as

$$
\Delta l^{2}=\frac{1}{T} \Delta T \Delta S-\frac{1}{T} \Delta P \Delta V .
$$

To express the above line element in $(S, P)$ coordinates, we have

$$
\begin{aligned}
& \Delta T=\left.\frac{\partial T}{\partial S}\right|_{P} \Delta S+\left.\frac{\partial T}{\partial P}\right|_{S} \Delta P, \\
& \Delta V=\left.\frac{\partial V}{\partial S}\right|_{P} \Delta S+\left.\frac{\partial V}{\partial P}\right|_{S} \Delta P .
\end{aligned}
$$

Substituting Eqs. (15) into Eq. (14) and using the Maxwell relation

$$
\left.\frac{\partial T}{\partial P}\right|_{S}=\left.\frac{\partial V}{\partial S}\right|_{P}
$$

one obtains the thermodynamic line element

$$
\Delta l^{2}=\frac{1}{C_{P}} \Delta S^{2}+\frac{V}{T} \kappa_{S} \Delta P^{2},
$$

where $C_{P}=T(\partial S / \partial T)_{P}$ is the heat capacity at constant pressure and $\kappa_{S}=-1 / V(\partial V / \partial P)_{S}$ is the adiabatic compressibility. Here, the thermodynamic potential is the enthalpy where the independent variables are entropy and pressure.

\section{B. Thermodynamic curvature in $\boldsymbol{P}-\boldsymbol{V}$ diagram}

Now, we use Eq. (16) to investigate microstructure of $\mathrm{RN}-\mathrm{AdS}$ black hole in an extended phase space. Because of the fact that the entropy of black hole only depends on the volume, i.e., $S=\left(3^{2} \pi / 2^{4}\right)^{1 / 3} V^{2 / 3}$, the line element of the Ruppeiner geometry can be written as

$$
\Delta l^{2}=\frac{1}{C_{P}}\left(\frac{\pi}{6 V}\right)^{2 / 3} \Delta V^{2}+\frac{V}{T} \kappa_{S} \Delta P^{2}
$$

where the pressure and volume are taken as the fluctuation variables. For the black hole, the adiabatic compressibility $\left(\kappa_{S}\right)$ vanishes similarly to the heat capacity at constant volume, i.e., $C_{V}=T(\partial S / \partial T)_{V}=0 .{ }^{1}$ Hence, following Refs. [38,39], we define a normalized thermodynamic curvature, $R_{N}$, based on the adiabatic compressibility

$$
R_{N}=R \kappa_{S}
$$

In what follows, we analyze in detail the behavior of the normalized thermodynamic curvature as function of the pressure and volume. By performing simple calculations, we obtain the normalized thermodynamic curvature

$R_{N}=\frac{16 \tilde{V}^{2 / 3}\left(3 \tilde{V}^{2 / 3}-1\right)\left(5-6 \tilde{V}^{2 / 3}+9 \tilde{P} \tilde{V}^{4 / 3}\right)}{\left(1-2 \tilde{V}^{2 / 3}+\tilde{P} \tilde{V}^{4 / 3}\right)^{2}\left(1-6 \tilde{V}^{2 / 3}-3 \tilde{P} \tilde{V}^{4 / 3}\right)}$,

which is expressed in terms of the reduced thermodynamic variables. Remarkably, the $R_{N}$ is independent of the charge of a black hole in Eq. (19). It should be noted that if one uses Eq. (16) instead of Eq. (17) for the Ruppeiner line element the normalized thermodynamic curvature $\left(R_{N}\right)$, Eq. (19), does not change. The overall behavior of the normalized thermodynamic curvature as a function of $P / P_{c}$ and $V / V_{c}$ is illustrated in Fig. 2. As can be ascertained from Fig. 2, the $R_{N}$ goes to negative infinity in certain regions of the plane. From Eq. (19), $R_{N}$ diverges along the curves

$$
\begin{gathered}
\tilde{P}_{\mathrm{div}}=\frac{2 \tilde{V}^{2 / 3}-1}{\tilde{V}^{4 / 3}}, \\
\tilde{P}_{\mathrm{div}}=\frac{1-6 \tilde{V}^{2 / 3}}{3 \tilde{V}^{4 / 3}} .
\end{gathered}
$$

The divergent curve in Eq. (21) corresponds to the extremal black holes, which are at zero temperature. On the other hand, $R_{N}$ obviously vanishes at the curves

\footnotetext{
${ }^{1}$ The entropy of the van der Waals fluid system is a function of the temperature and volume i.e., $S=S(T, V)[39,46]$. This would imply that the adiabatic compressibility is nonzero $\left(\kappa_{S} \neq 0\right)$ and it has a finite value at the critical point.
} 


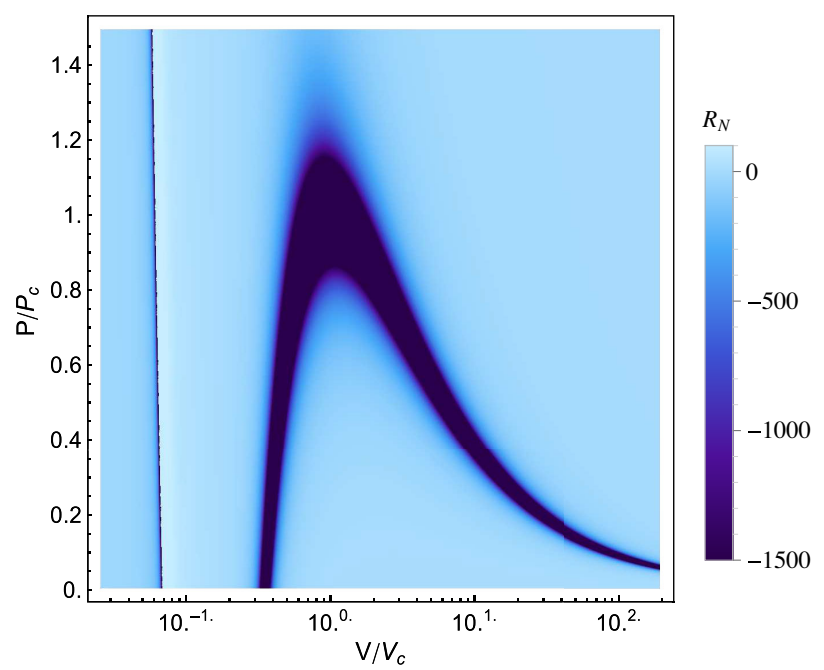

FIG. 2. The normalized thermodynamic curvature as a function of the pressure $P$ and volume $V$. Note the logarithmic scale on the horizontal axis.

$$
\begin{aligned}
& \tilde{P}_{0}=\frac{6 \tilde{V}^{2 / 3}-5}{9 \tilde{V}^{4 / 3}}, \\
& \tilde{V}_{0}=\frac{1}{3 \sqrt{3}},
\end{aligned}
$$

where the dominant interaction between the microstructure of charged black hole changes from attractive to repulsive and vice versa.

To better understand the behavior of the normalized thermodynamic curvature, we show the diverging (gray dashed line) and vanishing (brown dotted line) curves corresponding to Eqs. (20) and (21) and Eq. (22), respectively, as well as the small-large black hole phase transition (light blue solid line) curve in Fig. 3. In Fig. 3, the critical point is highlighted by a black solid circle, and the shaded regions have positive values for $R_{N}$ which imply the domination of repulsive interaction. In the other region, $R_{N}$ is negative, which means the microstructure interactions are attractive. As evident from Fig. $3, R_{N}$ is negative for the large black hole, while there is a certain range of volume in the small black hole phase $(\tilde{V}<1)$ that has positive $R_{N}$. In this positive region, $R_{N}$ diverges to positive infinity when the gray dashed line is approached from large values of volume; i.e., the microstructure interaction of the small black hole is strongly repulsive. A strongly repulsive interaction also exists in the higher pressure regime (above the critical point) at low volume $\tilde{V}$. The white region to the left of the gray dashed curve on the left side of the Fig. 3, where black holes are sufficiently small, is excluded due to the fact that temperature is negative. Since the equation of state (8) may not hold in the transition region (below the light blue solid curve), $R_{N}$ does not give any information about the black hole microstructure. Furthermore, as also seen in Fig. 3, light blue solid and gray dashed curves

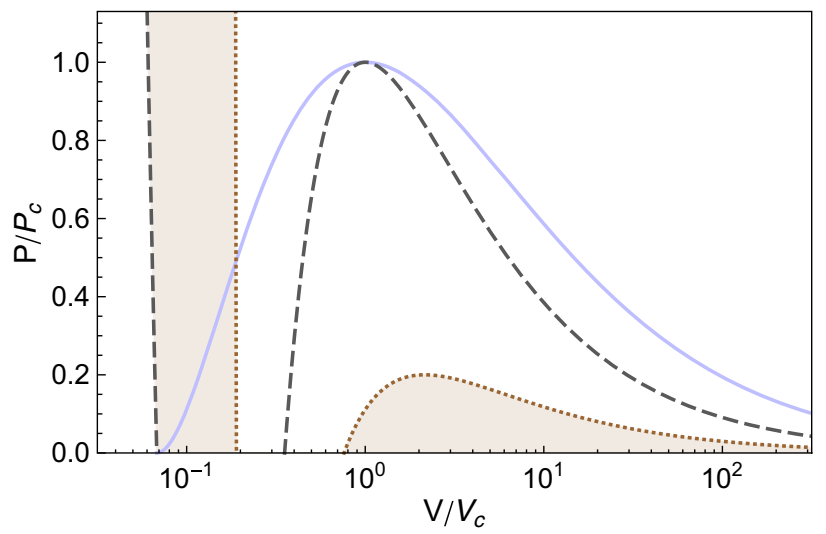

FIG. 3. Transition curve (light blue solid line), vanishing curve (brown dotted line) and diverging curve (gray dashed line) of $R_{N}$. The region identified corresponds to positive $R_{N}$; otherwise, $R_{N}$ is negative. Both the transition and diverging curves start at $\tilde{V}=1 / 6 \sqrt{6}$. The region to the left of the shaded region on the left side of the figure is excluded because temperature is negative. The critical point is marked by a black spot. Note the logarithmic scale on the horizontal axis.

coincide at the critical point where the thermodynamic functions of charged black hole are characterized by a set of critical exponents [4]. Hence, the normalized thermodynamic curvature diverges to negative infinity $\left(R_{N} \rightarrow-\infty\right)$ at the critical point. This situation is analogous to fluid in the critical point regime, such as the van der Waals system $[30,38,39]$, where thermodynamic curvature goes to negative infinity at the critical point.

To obtain an explicit expression of $R_{N}$ near the critical point, we expand $R_{N}$, Eq. (19), around the critical point using Eq. (8),

$$
R_{N}=-\frac{9}{2} t^{-2}
$$

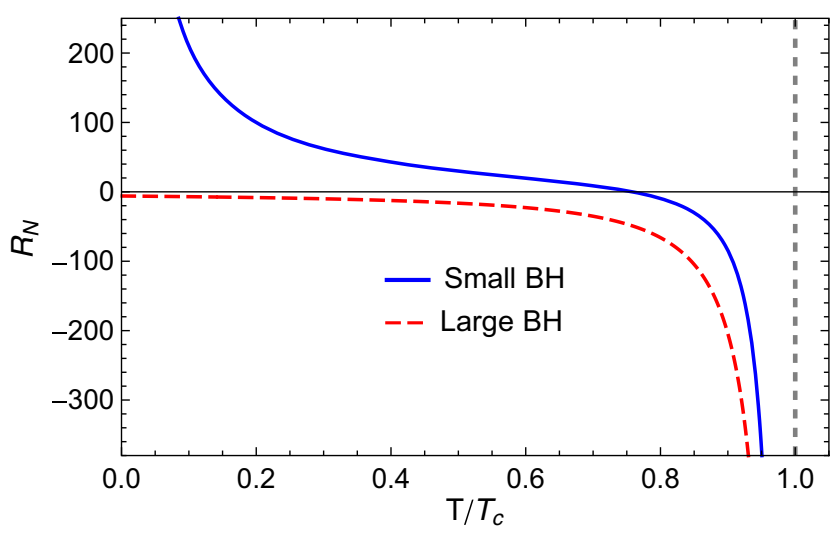

FIG. 4. The normalized thermodynamic curvature $R_{N}$ for charged AdS black hole along the transition curve in the small and large black holes phases. $R_{N}$ of the small black hole changes the sign to positive at $\tilde{T}=3 \sqrt{3}(7-3 \sqrt{5}) / 2 \approx 0.7581$. 
where $t=1-\tilde{T}$ is the deviation from the critical temperature. Therefore, $R_{N}$ has the universal critical exponent 2 and critical amplitude $-9 / 2$. Further, it is interesting to investigate the behavior of $R_{N}$ on the transition curve. In this respect, we plotted in Fig. $4 R_{N}$ along the transition curve in both the small and the large black hole phases from the critical temperature to zero. One observes from Fig. 4 that $R_{N}$ in both phases diverges to $-\infty$ at the critical temperature. In the large black hole phase, $R_{N}$ uniformly negative, and $\left|R_{N}\right|$ decreases as the temperature decreases from the critical temperature, and $R_{N}$ is small at $\tilde{T}=0$, while in the small black hole phase, $R_{N}$ changes sign and becomes positive below $\tilde{T}=3 \sqrt{3}(7-3 \sqrt{5}) / 2 \approx 0.7581$. Remarkably, $R_{N}$ diverges to positive infinity as $\tilde{T}$ tends to zero where strong repulsive interactions dominate.

\section{CONCLUSIONS}

In this paper, we proposed a new thermodynamic curvature, by using the adiabatic compressibility, for examining the internal microstructure of charged AdS black holes in an extended phase space. We explored the microscopic properties of small-large black holes phase transition by considering the pressure and volume as the fluctuation variables.
We defined a normalized thermodynamic curvature, $R_{N}=$ $\kappa_{S} R$, where $\kappa_{S}$ is the adiabatic compressibility, and studied the behavior of $R_{N}$ as a function of the pressure and volume. The sign of $R_{N}$ determines the repulsive or attractive feature of black holes microstructure. When $R_{N}>0$, the repulsive interaction dominates, while $R_{N}<0$ indicates that the microstructure interactions are attractive. We also observed that a strongly repulsive interaction exists in the higher pressure regime (above the critical point) at low volume. At the critical point, however, we have $R_{N} \rightarrow-\infty$, which is analogous to the van der Waals fluid in its critical point regime.

\section{ACKNOWLEDGMENTS}

We are grateful to the Research Council of Shiraz University. The work of A. S. has been financially supported by the Research Institute for Astronomy \& Astrophysics of Maragha (RIAAM), Iran. A. S. thanks Hermann Nicolai and Max-Planck-Institute for Gravitational Physics for hospitality. This work was supported by the National Natural Science Foundation of China (Grant No. 11675064) and the Fundamental Research Funds for the Central Universities (Grant No. lzujbky-2019-it21).
[1] S. Hawking and D. N. Page, Thermodynamics of black holes in anti-de sitter space, Commun. Math. Phys. 87, 577 (1983).

[2] E. Witten, Anti-de Sitter space, thermal phase transition, and confinement in gauge theories, Adv. Theor. Math. Phys. 2, 505 (1998).

[3] B. P. Dolan, The cosmological constant and the black hole equation of state, Classical Quantum Gravity 28, 125020 (2011); Pressure and volume in the first law of black hole thermodynamics, Classical Quantum Gravity 28, 235017 (2011).

[4] D. Kubiznak and R. B. Mann, P-V criticality of charged AdS black holes, J. High Energy Phys. 07 (2012) 033.

[5] D. Kastor, S. Ray, and J. Traschen, Enthalpy and the mechanics of AdS black holes, Classical Quantum Gravity 26, 195011 (2009).

[6] S. H. Hendi and M. H. Vahidinia, Extended phase space thermodynamics and P-V criticality of black holes with a nonlinear source, Phys. Rev. D 88, 084045 (2013).

[7] M. B. Jahani Poshteh, B. Mirza, and Z. Sherkatghanad, Phase transition, critical behavior, and critical exponents of Myers-Perry black holes, Phys. Rev. D 88, 024005 (2013).

[8] Z. Sherkatghanad, B. Mirza, Z. Mirzaeyan, and S. A. H. Mansoori, Critical behaviors and phase transitions of black holes in higher order gravities and extended phase spaces, Int. J. Mod. Phys. D 26, 1750017 (2017).
[9] R. Banerjee and D. Roychowdhury, Critical phenomena in Born-Infeld AdS black holes, Phys. Rev. D 85, 044040 (2012); Critical behavior of Born Infeld AdS black holes in higher dimensions, Phys. Rev. D 85, 104043 (2012).

[10] M. H. Dehghani, S. Kamrani, and A. Sheykhi, P-V criticality of charged dilatonic black holes, Phys. Rev. D 90, 104020 (2014).

[11] M. H. Dehghani, A. Sheykhi, and Z. Dayyani, Critical behavior of Born-Infeld dilaton black holes, Phys. Rev. D 93, 024022 (2016).

[12] Z. Dayyani, A. Sheykhi, and M. H. Dehghani, Counterterm method in dilaton gravity and the critical behavior of dilaton black holes with power-Maxwell field, Phys. Rev. D 95, 084004 (2017).

[13] Z. Dayyani, A. Sheykhi, M. H. Dehghani, and S. Hajkhalili, Critical behavior and phase transition of dilaton black holes with nonlinear electrodynamics, Eur. Phys. J. C 78, 152 (2018).

[14] A. Dehghani and S. H. Hendi, Charged black hole chemistry with massive gravitons, Classical Quantum Gravity 37, 024001 (2020).

[15] S. Gunasekaran, R. B. Mann, and D. Kubiznak, Extended phase space thermodynamics for charged and rotating black holes and Born-Infeld vacuum polarization, J. High Energy Phys. 11 (2012) 110. 
[16] A. Dehyadegari, A. Sheykhi, and A. Montakhab, Novel phase transition in charged dilaton black holes, Phys. Rev. D 96, 084012 (2017).

[17] N. Altamirano, D. Kubiznak, and R. B. Mann, Reentrant phase transitions in rotating anti-de Sitter black holes, Phys. Rev. D 88, 101502 (2013).

[18] S.-W. Wei and Y.-X. Liu, Triple points and phase diagrams in the extended phase space of charged Gauss-Bonnet black holes in AdS space, Phys. Rev. D 90, 044057 (2014).

[19] R. A. Hennigar, R. B. Mann, and E. Tjoa, Superfluid Black Holes, Phys. Rev. Lett. 118, 021301 (2017).

[20] B. R. Majhi and S. Samanta, $P-V$ criticality of AdS black holes in a general framework, Phys. Lett. B 773, 203 (2017).

[21] A. Chamblin, R. Emparan, C. V. Johnson, and R. C. Myers, Charged AdS black holes and catastrophic holography, Phys. Rev. D 60, 064018 (1999); Holography, thermodynamics and fluctuations of charged AdS black holes, Phys. Rev. D 60, 104026 (1999).

[22] A. Dehyadegari, A. Sheykhi, and A. Montakhab, Critical behaviour and microscopic structure of charged AdS black holes via an alternative phase space, Phys. Lett. B 768, 235 (2017).

[23] A. Dehyadegari, B. R. Majhi, A. Sheykhi, and A. Montakhab, Universality class of alternative phase space and van der Waals criticality, Phys. Lett. B 791, 30 (2019).

[24] Z. Dayyani and A. Sheykhi, Critical behavior of Lifshitz dilaton black holes, Phys. Rev. D 98, 104026 (2018).

[25] H. Yazdikarimi, A. Sheykhi, and Z. Dayyani, Critical behavior of Gauss-Bonnet black holes via an alternative phase space, Phys. Rev. D 99, 124017 (2019).

[26] A. Sheykhi, M. Arab, Z. Dayyani, and A. Dehyadegari, Alternative approach towards critical behavior and microscopic structure of the higher dimensional Power-Maxwell black holes, Phys. Rev. D 101, 064019 (2020).

[27] A. Dehyadegari and A. Sheykhi, Reentrant phase transition of Born-Infeld-AdS black holes, Phys. Rev. D 98, 024011 (2018).

[28] A. Dehyadegari and A. Sheykhi, Critical behavior of charged dilaton black holes in AdS space, Phys. Rev. D 102, 064021 (2020).

[29] G. Ruppeiner, Thermodynamics: A Riemannian geometric model, Phys. Rev. A 20, 1608 (1979).

[30] G. Ruppeiner, Riemannian geometry in thermodynamic fluctuation theory, Rev. Mod. Phys. 67, 605 (1995); 68, 313 (1996).
[31] G. Ruppeiner, Thermodynamic curvature measures interactions, Am. J. Phys. 78, 1170 (2010).

[32] G. Ruppeiner, Thermodynamic curvature from the critical point to the triple point, Phys. Rev. E 86, 021130 (2012).

[33] H. O. May, P. Mausbach, and G. Ruppeiner, Thermodynamic curvature for attractive and repulsive intermolecular forces, Phys. Rev. E 88, 032123 (2013).

[34] G. Ruppeiner, Thermodynamic curvature: Pure fluids to black holes, J. Phys. Conf. Ser. 410, 012138 (2013).

[35] G. Ruppeiner, Thermodynamic curvature and black holes, Springer Proc. Phys. 153, 179 (2014).

[36] S.-W. Wei and Y.-X. Liu, Insight Into the Microscopic Structure of an AdS Black Hole from Thermodynamical Phase Transition, Phys. Rev. Lett. 115, 111302 (2015); Erratum, Phys. Rev. Lett. 116, 169903 (2016).

[37] M. Kord Zangeneh, A. Dehyadegari, and A. Sheykhi, Comment on "Insight into the Microscopic Structure of an AdS Black Hole from a Thermodynamical Phase Transition", arXiv:1602.03711.

[38] S.-W. Wei, Y.-X. Liu, and R. B. Mann, Repulsive Interactions and Universal Properties of Charged AdS Black Hole Microstructures, Phys. Rev. Lett. 123, 071103 (2019).

[39] S.-W. Wei, Y.-X. Liu, and R. B. Mann, Ruppeiner geometry, phase transitions, and the microstructure of charged AdS black holes, Phys. Rev. D 100, 124033 (2019).

[40] S.-W. Wei and Y.-X. Liu, New insights into thermodynamics and microstructure of AdS black holes, Sci. Bull. 65, 259 (2020).

[41] Z.-M. Xu, B. Wu, and W.-L. Yang, Ruppeiner thermodynamic geometry for the Schwarzschild AdS black hole, Phys. Rev. D 101, 024018 (2020).

[42] A. Ghosh and C. Bhamidipati, Thermodynamic geometry for charged Gauss-Bonnet black holes in AdS spacetimes, Phys. Rev. D 101, 046005 (2020).

[43] A. Ghosh and C. Bhamidipati, Thermodynamic geometry and interacting microstructures of BTZ black holes, Phys. Rev. D 101, 106007 (2020).

[44] S Mahish, A. Ghosh, and C. Bhamidipati, Chemical potential, microstructures and phase transition of the Schwarzschild-AdS black hole, arXiv:2006.02943.

[45] E. Spallucci and A. Smailagic, Maxwell's equal area law for charged Anti-de Sitter black holes, Phys. Lett. B 723, 436 (2013).

[46] L. D. Landau and E. M. Lifzhitz, Statistical Physics (Pergamon, New York, 1977). 